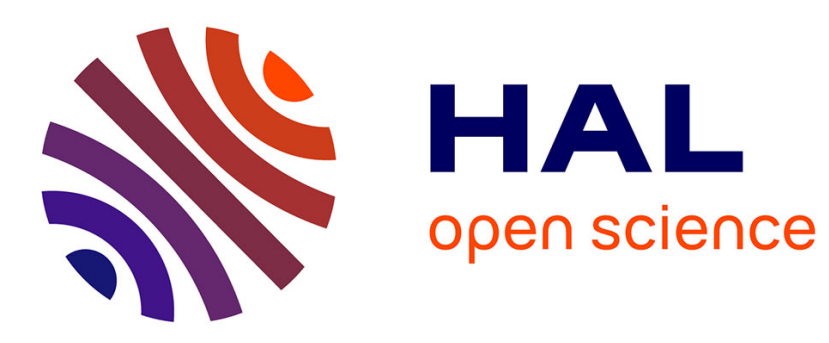

\title{
An independent loops search algorithm for solving inductive PEEC large problems
}

Trung Son Nguyen, Jean-Michel Guichon, Olivier Chadebec, Gérard Meunier, Benjamin Vincent

\section{- To cite this version:}

Trung Son Nguyen, Jean-Michel Guichon, Olivier Chadebec, Gérard Meunier, Benjamin Vincent. An independent loops search algorithm for solving inductive PEEC large problems. Progress In Electromagnetics Research M, 2012, 23, pp.53-63. 10.2528/PIERM11111503 . hal-00727824

\section{HAL Id: hal-00727824 \\ https://hal.science/hal-00727824}

Submitted on 2 Feb 2021

HAL is a multi-disciplinary open access archive for the deposit and dissemination of scientific research documents, whether they are published or not. The documents may come from teaching and research institutions in France or abroad, or from public or private research centers.
L'archive ouverte pluridisciplinaire HAL, est destinée au dépôt et à la diffusion de documents scientifiques de niveau recherche, publiés ou non, émanant des établissements d'enseignement et de recherche français ou étrangers, des laboratoires publics ou privés. 


\title{
AN INDEPENDENT LOOPS SEARCH ALGORITHM FOR SOLVING INDUCTIVE PEEC LARGE PROBLEMS
}

\author{
T.-S. Nguyen*, J.-M. Guichon, O. Chadebec, G. Meunier, \\ and B. Vincent
}

Grenoble Electrical Engineering Laboratory, Grenoble-INP, Université Joseph Fourier, CNRS UMR 5269, Grenoble, France

\begin{abstract}
This paper describes an original approach for determining independent loops needed for mesh-current analysis in order to solve circuit equation system arising in inductive Partial Element Equivalent Circuit (PEEC) approach. Based on the combined used of several simple algorithms, it considerably speed-up the loops search and enables the building of an associated matrix system with an improved condition number. The approach is so well-suited for large degrees of freedom problems, saving significantly memory and decreasing the time of resolution.
\end{abstract}

\section{INTRODUCTION}

Partial element equivalent circuit (PEEC) approach [1] is known to be very suitable for the modeling of combined circuit and electromagnetic field problems. For low frequency applications, capacitive effects can be neglected and the method can be restricted to its inductive formulation [2]. With such approach, arbitrarily shaped 3D conducting devices can be represented by equivalent circuits combining resistors, partial/mutual inductances and current and/or voltage sources. The circuit-based model of electromagnetic devices makes its interface with SPICE-like circuit solvers possible. The approach is known to be very efficient for the electromagnetic modeling of power electronic modules for instance [2].

In order to solve circuit equations obtained by PEEC approach, node-voltage and mesh-current analysis are two well-known methods which allow the circuit analysis. For circuits with many mutual

* Corresponding author: Trung-Son Nguyen (Trung-Son.NGUYEN@g2elab.grenobleinp.fr). 
inductances such as those provided here, nodal analysis (based on standard Kirchhoff's equations) is known to be unsuitable, leading to a higher degrees of freedom and to a matrix system with a bad condition number. On the other hand, the use of independent loops - current analysis (based on independent loop search) could reduce significantly the number of circuit equations and therefore leads to fewer unknowns and also provides a much better condition number for the system [3].

Despite this clear advantage, this approach requires to identify a set of independent loops, which is well known to be an uneasy task especially in large problems. Many strategies has been proposed to determine these independent loops, such as general algebraic matrix solution by inspection of the circuit [4] or graph algorithms $[5,6]$. Method proposed in [4] is general but extremely time and memory consuming for 3D structures meshed with a high density. Recently, a new method [6] has been proposed and shown more efficient than the generic graph solution presented in [5].

In this paper, an alternative novel strategy coupling a general loop matrix method with a simple graph algorithm is proposed. The method is a generic circuit solution such as $[4,5]$ and requires a similar computational complexity than [6]. From numerical point of view, it leads to a reliable set of equations leading to a matrix system with most important terms located mainly close to the diagonal. Therefore, the convergence of iterative linear solvers is improved. Let us notice that this method is also compliant with the use of Adaptative Multi-Level Fast Multipole Method (AMLFMM) [7] which improved the speed of the algorithm. An industrial example meshed with more than ten thousands branches has been solved with this approach.

\section{INDUCTIVE PEEC METHOD}

\subsection{Equivalent Circuit Formulation}

Let us consider $N_{b}$ volume conductors fed with alternative sources placed in a surrounding air region without any magnetic materials. PEEC method is based on the determination of partial voltage generated on each conductor by electromagnetic sources. To compute these voltages, volume integration on the considered conductor of the magnetic vector potential created by all the others conductor is provided [1]. Let us assume that the current density in each conductor is uniform. The expression of the magnetic vector potential $\mathbf{A}_{j}$ created by conductor $j$ is:

$$
\mathbf{A}_{j}(P)=\frac{\mu_{0}}{4 \pi} \frac{I_{j}}{S_{j}} \int_{V_{j}} \frac{\mathbf{l}_{j}}{r} d V_{j}
$$


where $r$ is the distance between the integration point and the point $P, I_{j}$ is the current in the conductor $j, S_{j}$ is the cross-section of the current flow and $V_{j}$ is the conductor volume and $\mathbf{l}_{j}$ is the unit vector oriented in the direction of current $I_{j}$. The flux created by $I_{j}$ through the conductor $k$ is calculated with:

$$
\Phi_{k j}=\frac{1}{S_{k}} \int_{V_{k}} \mathbf{A}_{j} \cdot \mathbf{l}_{k} d V_{k}=\frac{\mu_{0}}{4 \pi} \frac{I_{j}}{S_{j} S_{k}} \int_{V_{k}} \int_{V_{j}} \frac{\mathbf{l}_{j} \mathbf{l}_{k}}{r} d V_{j} d V_{k}
$$

The mutual inductance between the two conductors is defined by:

$$
m_{k j}=\frac{\Phi_{k j}}{I_{j}}=\frac{\mu_{0}}{4 \pi} \frac{1}{S_{j} S_{k}} \int_{V_{k}} \int_{V_{j}} \frac{\mathbf{l}_{j} \mathbf{l}_{k}}{r} d V_{j} d V_{k}
$$

The partial mutual inductance $m_{k j}$ only depends on the dimensions and the positions of conductor $k$ and $j$ and can be calculated by analytic formulas or numerical procedures. Thus, the voltage appearing on the conductor $k$ is given by:

$$
U_{k}=I_{k} \cdot R_{k}+j \omega \sum_{j=1}^{N_{b}} I_{j} m_{k j}
$$

where $\omega$ is the angular frequency, $R_{k}$ is the DC resistance of $k$-th conductor. Equation (4) links partial voltages of conductors to currents flowing in them. It must be pointed out that this approach can also be generalized for the modeling of bi-directional conductors (conductive plates), representing them by a grid of unidirectional perpendicular conductors in order to create orthogonal bases for currents.

\subsection{Independent Loop Analysis}

If the current flowing into each elementary conductor (or branch) is $\mathbf{I}_{b}$ and associated voltage is $\mathbf{V}_{b}$, we get a system of equations linking currents vector to voltages vector.

$$
\mathbf{Z}_{b} \mathbf{I}_{b}=\left(\mathbf{R}_{b}+j \omega \mathbf{L}_{b}\right) \mathbf{I}_{b}=\mathbf{V}_{b}
$$

where $\mathbf{Z}_{b}$ is called branch impedance matrix, $\mathbf{R}_{b}$ is a diagonal matrix and $\mathbf{L}_{b}$ is a fully dense matrix of partial inductances. These matrices are square and have $N_{b} \times N_{b}$ elements.

In order to solve the problem, it remains to add circuit equations (Kirchhoff's circuit law) ensuring the current conservation. Using an independent loop analysis in the graph representing the circuit, we can transform (5) into a new equations system:

$$
\mathbf{M Z}_{b} \mathbf{M}^{t} \mathbf{I}_{m}=\mathbf{Z}_{m} \mathbf{I}_{m}=\mathbf{M} \mathbf{V}_{b}=\mathbf{V}_{m}
$$


where $\mathbf{I}_{m}$ is an independent loops-based current vector $\left(\mathbf{M}^{t} \mathbf{I}_{m}=\right.$ $\mathbf{I}_{b}$ ), matrix $\mathbf{M}$ is the branches/independent loops transition matrix (populated by $-1,0$ or 1 ), $\mathbf{Z}_{m}$ is an independent loop-based impedance matrix and $\mathbf{V}_{m}$ is the vector of source voltages (most part of vector equal to 0). Let us notice that one loop may be composed of meshed PEEC elements and external electric component. In this approach, unknowns are independent loop current $\mathbf{I}_{m}$ and their number are reduced (the number of loops being less important than the number of conductors). It leads to a better condition number in comparison with nodal analysis [3].

\subsection{Introduction of Compression Algorithm}

Matrix $\mathbf{L}_{b}$, as well as $\mathbf{L}_{m}$, is fully dense. It leads to a prohibitive time of computation and memory storage if problem with industrial complexity are considered. The coupling with a compression algorithm is then needed. The introduction of "Adaptative Multi-Level Fast Multipole Method" (AMLFMM) in the solving process leads to the storage of the $\mathbf{Z}_{b}$ part corresponding to near field interactions whereas the far field interactions are compressed thanks to multipole expansions [7]. As a consequence, the matrix $\mathbf{Z}_{b}$ is split into a near-field matrix (still fully dense but really smaller) and a far-field compression:

$$
\mathbf{Z}_{b}=\mathbf{Z}_{b_{-} \text {near }}+\mathbf{Z}_{b_{\text {_far }}}
$$

Let us notice that to compute the $\mathbf{Z}_{m} \cdot \mathbf{I}_{m}$ product (needed in the iterative solving process), (7) is simply multiplied by $\mathbf{M}$ transition sparse matrix and $\mathbf{I}_{m}$ current. In order to ensure the convergence of the solving process, a preconditioning technique using a partial $\mathrm{LU}$ decomposition of $\mathbf{Z}_{m \_n e a r}$ is applied [8].

\section{FUNDAMENTAL LOOPS IDENTIFICATION ALGORITHM}

From this section, we will explain how to identifier matrix $\mathbf{M}$ in the Equation (6) of large systems from the global circuit of PEEC method. In order to make it easier to understand, we introduce some simple graph theory which considerer the PEEC global circuit as an initial undirected connected graph.

\subsection{Basic Graph Theory for Independent Loops Identification}

The global graph is composed of many independents loops. A subcircuit can be defined as a set of independent loops related to each 


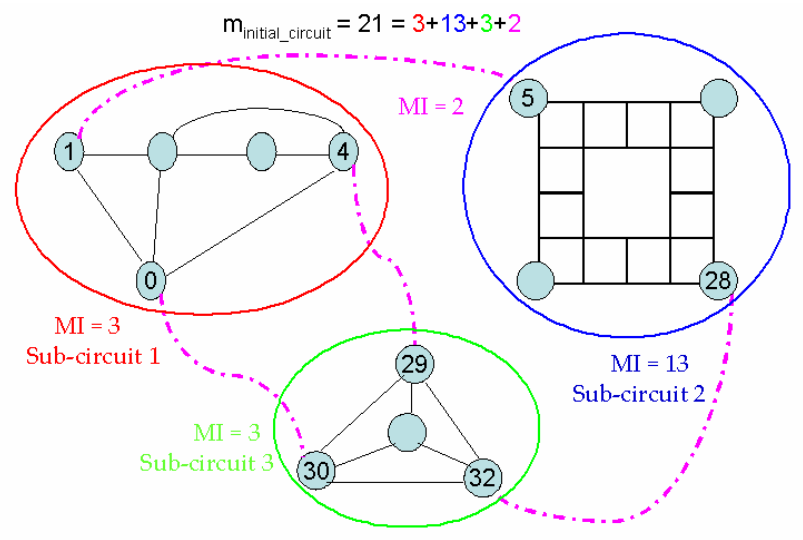

Figure 1. Union of three set of loops (sub-circuits in red, blue and green) in PEEC network. Super-circuit appears in purple.

other. The surrounding circuit connecting a set of sub-circuits between each other will be called super-circuit (the rest of initial circuit except all sub-circuit). It is also composed of independent loops. The initial circuit is then composed of a set of sub-circuits and a super-circuit. The union of these both sets of independent loops is the complete set of independent loops (see Figure 1). Therefore if $m$ denotes an independent loop, we get:

$$
m_{\text {initial_circuit }}=m_{\text {super_circuit }}+\sum_{\text {all_sub_circuit }} m_{\text {sub_circuit }}
$$

Keep these considerations in mind; we can propose a strategy to identify all of fundamental loops:

Step 1: Partition initial circuit into several sub-circuits and detects all fundamental loops in each of them.

Step 2: Create the super-circuit from initial circuit and subcircuits and then search all fundamental loops in it.

In the case of nontrivial graphs (multiple connected domains), each connected graph becomes an initial circuit and the total number of independent loops is the sum of all connected graphs. We get:

$$
\begin{aligned}
m_{\text {multiple_connected_domains }}= & \sum_{\text {all_connected_domain }} m_{\text {each_domain }} \\
m_{\text {all }}= & \sum_{\text {all_connected_domains }} m_{\text {super_circuit }} \\
& +\sum_{\text {all_connected_domains }}\left(\sum_{\text {all_sub_circuit }} m_{\text {sub_circuit }}\right)
\end{aligned}
$$




\subsection{Sub-circuit Determination and Independent Loop Search}

In order to search fundamental loops, we propose the following algorithm (see Figure 2).

Step 1a: If we have no idea about the number of branches in a fundamental loop, a general method [4] can be used but remains very expensive in term of computation time. On the other hand, a very efficient way which speeds-up the algorithm is to focus on the search of independent loops with at most $k$ branches. In common applications, surface conductors are under study. The typical PEEC discretization is then mainly composed by a grid of bi-directional inductances (see

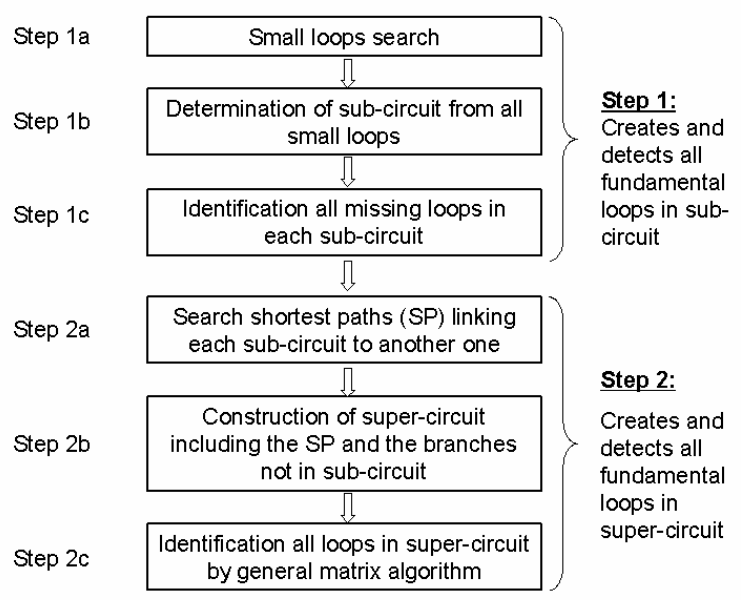

Figure 2. Principle of independent loops search algorithm. Six different steps are proposed.

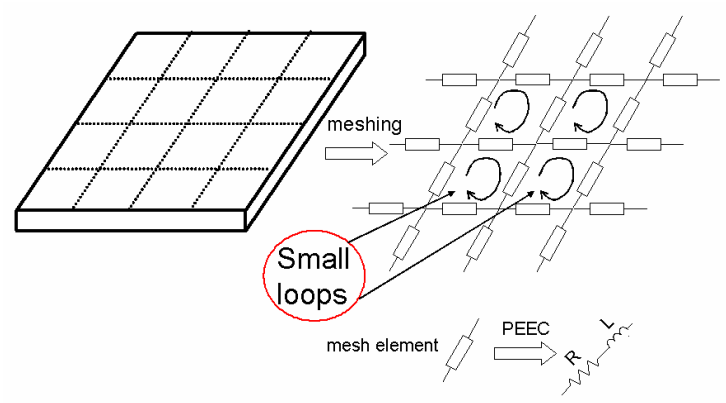

Figure 3. Example of some small loops detected on a standard PEEC conductor mesh. 
Figure 3), with obvious small independent loops of 4 conductors. The number $k=4$ is then chosen to ensure a partial but very quick detection of the largest number of loops as possible. The search is based on a classical graph search algorithm but limited to the detection of obvious solutions. More complex loops will be treated in a future step in order to reduce the algorithm complexity.

Step 1b: From the set of small loops get in Step 1a, it remains to create the set of sub-circuit. In order to create a sub-circuit, we propose to aggregate all the small independent loops which share at least one branch. This process is illustrated in Figure 4(a), where we see that all small loops are grouped to create a sub-circuit. However, the graph search algorithm being not complete, some loops can miss. In Figure 4(a), according to formula:

$m_{\text {sub_circuit }}=b-n+1=36-24+1=13,13$ independent loops are needed but only 12 small loops have been obtained ( $b$ branches and $n$ nodes attached to this sub-circuit). One fundamental loop in the sub-circuit is still missing. It remains to identify it. This is the purpose of the next algorithm step.

Step 1c: To get missing loops, a graph algorithm (minimal spanning tree) is used to find larger loops (with more than $k$ branches) in the sub-circuit. Let us go back to our example. In Figure 4(b), a sub-circuit divided into spanning-tree, incomplete co-tree and missing co-tree is presented. Each considered branch is included in spanningtree if this branch is not already included in it and if both attached

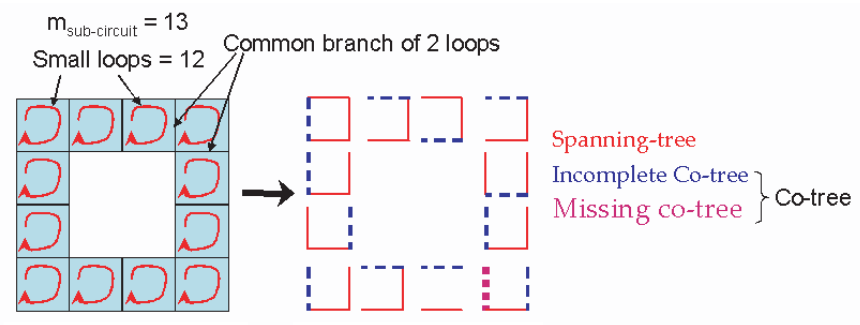

(a)

(b)
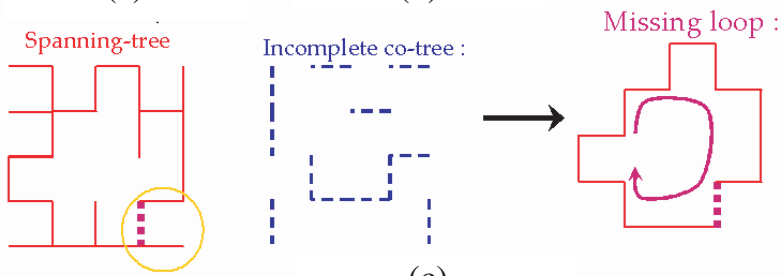

(c)

Figure 4. Sub-circuit loops analysis and detection of missing loops. (a) Sub-circuit created from small loops. (b) Graph of sub-circuit. (c) Search of missing loop. 
nodes (there are only two nodes per branch) are not already included in this list. Since co-tree list contained only one branch from each small independent loop, the violet branch in Figure 4(b) is added in the missing co-tree list. This point is confirmed by the fact that there is a last independent loop with the union of the spanning tree list and this branch as illustrated in Figure 4(c). A Breath First Search (BFS) algorithm is used in order to find the shortest path between the two nodes attached to this missing co-tree branch. After Step 1, all independent loops of sub-circuits in all connected domains have been found.

\subsection{Super-circuit Main Loop Analysis}

In Step 2a, we need to search a sub-circuit internal path linking its connections with the super-circuit (see Figure 5). This task is also achieved thanks to a BFS algorithm like the one used in Step 1c. At the end of the Step 2b, branches have been included in a list which composed of the sub-circuit internal path and the branches which have been not included in sub-circuits. This list defines the super-circuit, even if those branches belong to several multiple connected domains. Since this circuit has been reduced, the general matrix solution [4] is efficient to identify the last fundamentals loops (Step 2c).

\subsection{Complexity of the Algorithm}

In Step 1a, because of very small size of loop (at most 4 branches), independent loops identification in sub-circuits requires $O(|N|)$ computational time ( $\mathrm{N}$ being the number of nodes in the graph). The creation of sub-circuit in Step 1b, BFS algorithm in Step 1c, requires $O(|N|)$ in worst configurations.

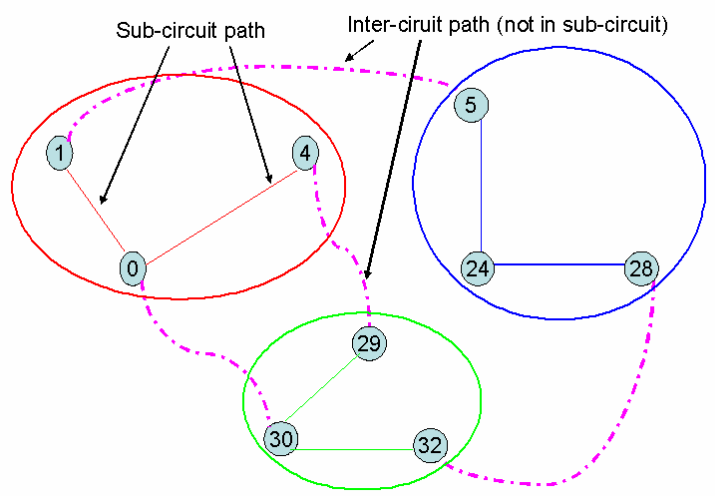

Figure 5. Reduction of general circuit to super-circuit. 
In Step 2a, another BFS algorithm is used. Step 2b just creates the super-circuit so both of them can not exceed $O(|N|)$ operations. The last task (Step 2c) is the most expensive in terms of complexity $\left(N^{3}\right)$ but if many sub-circuits have already been found, the complexity of this step remains the same but applied to a problem with a very few number independent loops. $N$ being small, the time needed for this step is not prohibitive (this is the case for typical inductive PEEC discretization).

\section{NUMERICAL RESULT}

Classical general matrix solution technique has been compared with this new approach. Both techniques have been implemented in InCa3D software [9]. We consider the modeling of a LED headlight PCB. The geometry is meshed with 11,445 branches and 4,762 fundamental loops have to be found (see Figure 6). An AMLFMM compression algorithm is used in order to speed-up the integration and the resolution process associated to iterative GMRES linear solver. To ensure the solving process convergence, a preconditioning technique is needed, as already mentioned in this paper; a partial LU decomposition of the near field impedance matrix is used [8]. The problem is solved on standard computer (PC Intel Core 2 Duo @2.66 GHz-2 GB memory).

In comparison with standard nodal analysis [3], the number of unknowns is considerably reduced (about 4.700 versus 18.000). Our approach considerably reduced the time needed for the search of independent loops in comparison with [4] (see Table 1).

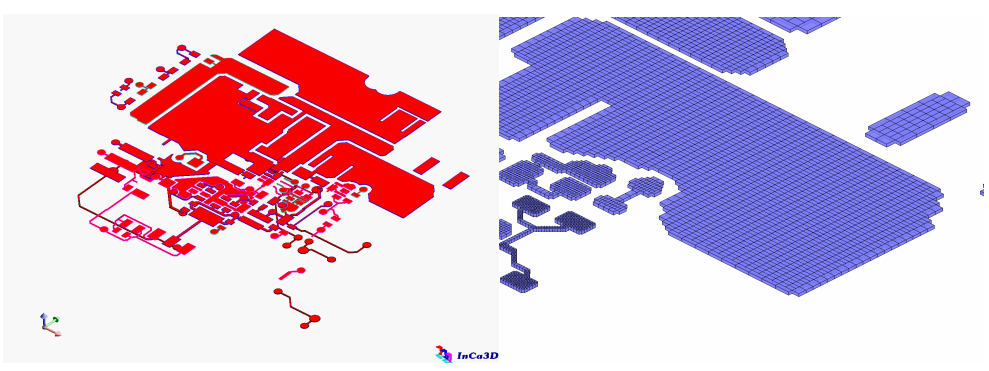

Figure 6. Car Headlight LED PCB modeling in InCa3D, general view of the geometry and mesh details (courtesy of Valeo).

Table 1. Time for determining a set of independent loop.

\begin{tabular}{|c|c|c|}
\hline & Classical general matrix solution [3] & New algorithm \\
\hline Time (s) & $2894 \mathrm{~s}$ & $2.1 \mathrm{~s}$ \\
\hline
\end{tabular}




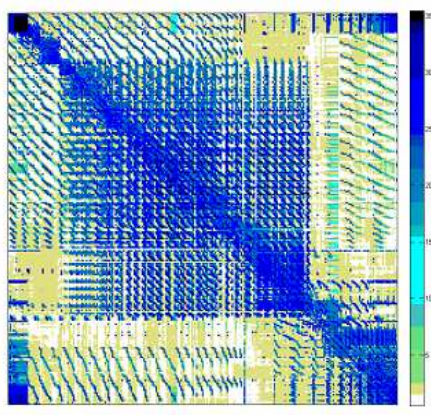

(a)

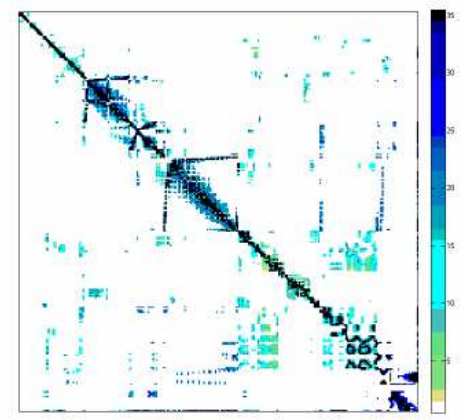

(b)

Figure 7. Structure of near-field interaction $Z_{m \_n e a r}$ matrix created by (a) general matrix solution and (b) new algorithm.

Another interesting aspect of our method is illustrated if we have a look to $Z_{m \_n e a r}$ (let us remember that in the real numerical process this matrix is never built, but it should give to the reader a good idea of terms repartition). Matrix get with our method and the one given by [4] are plot on Figure 7 (it must be pointed out that in this representation white terms - equal to 0 - are treated thanks to FMM). Let us remark that the matrix built thanks to our method presents a good concentration of higher element close to its diagonal. This positive structure facilitates the convergence of the iterative solver. Thus, using [4], 85 iterations are needed to get the solution with GMRES. Using our algorithm, only 39 iterations are necessary to obtain the solution with the same accuracy.

Additional information about computation times can be given. The total time for the current distribution computation is $85 \mathrm{~s} .2 .1 \mathrm{~s}$ are needed to analyze the circuit, $7.5 \mathrm{~s}$ to compute the near interactions and $75 \mathrm{~s}$ for the iterative solving process. The memory requirement does not exceed $240 \mathrm{MB}$.

\section{CONCLUSION}

This paper proposes a new algorithm to detect fundamental loops in inductive PEEC models. It is well-suited to treat large problems arising in industrial application. The proposed numerical scheme provides a very quick determination of a minimal number of unknowns and the matrix system obtained is better conditioned. Thus, the convergence of the solving process is improved. Its comparison with the new approach proposed in [6] could be an interesting perspective. 


\section{REFERENCES}

1. Ruehli, A. E., "Equivalent circuit models for three dimensional multiconductor systems," IEEE Trans. Microw. Theory Techn., Vol. 22, No. 3, 216-221, Mar. 1974.

2. Ardon, V., J. Aime, O. Chadebec, E. Clavel, J.-M. Guichon, and E. Vialardi, "EMC modeling of an industrial variable speed drive with an adapted PEEC method," IEEE Trans. Mag., Vol. 46, No. 8, 2892-2898, 2010.

3. Kamon, M. and J. R. Philips, "Preconditioning techniques for constrained vector potential integral equations, with application to 3-D magnetoquasistatic analysis of electronic packages," Proceedings of the Colorado Conference on Iterative Methods, Apr. 1994.

4. Pillage, L. T., et al., Electronic Circuit and System Simulation Methods, 371-381, McGraw-Hill Inc., 1995.

5. Rong, A., A. C. Cangellaris, and L. Dong, "A novel graph partitioning technique for enhancing the computational efficiency of the loop-tree generalized PEEC modeling of 3D interconnects," IEEE 14th Topical Meeting on Elect. Performance of Electron. Packaging, 253-256, 2005.

6. Antonini, G., D. Frigioni, and G. Miscione, "Hybrid formulation of the equation systems of the 3-D PEEC model based on graph algorithms," IEEE Trans. Circuits and Syst. - I: Reg. Papers, Vol. 57, No. 1, Jan. 2010.

7. Nguyen, T.-S., J.-M. Guichon, O. Chadebec, P. Labie, and J.L. Coulomb, "Ships magnetic anomaly computation with integral equation and fast multipole method," IEEE Trans. Mag., Vol. 47, No. 5, 1414-1417, 2011.

8. Nguyen, T.-S., J.-M. Guichon, O. Chadebec, G. Meunier, and T. Le-Duc, "Inner-outer preconditioning strategy for 3D inductance extraction coupling with fast multipole method," Proceedings of International Conference on Computation in Electromagnetics, Apr. 2011.

9. [Online] InCa3D Software, www.cedrat.com. 